\title{
LES COMMUNICATIONS par lumière visible : le Li-Fi
}

\author{
Jorge GARCIA-MARQUEZ \\ Suat TOPSU \\ Responsable de projets \\ européens Oledcomm \\ Fondateur et Président \\ d'Oledcomm, Vélizy- \\ Villacoublay, France \\ jorge.garcia@oledcomm.com \\ suat@oledcomm.com
}

L'arrivée de diodes électroluminescentes (LED) plus puissantes et plus rapides a permis de penser à leur utilisation comme des transmetteurs d'information. La lumière émise par les LED devient un canal de communication sans fil permettant des applications dans des espaces intérieurs et dans certains cas dans des espaces ouverts. Nous connaissons ce type de communications sous le nom de «Li-Fi».

électromagnétique. Les communications dans le spectre RF (radiofréquences) disposent d'une bande d'approximativement $300 \mathrm{GHz}$ alors que la bande du spectre visible est de $400 \mathrm{THz}$. De plus, puisque la lumière visible est spatialement très localisée, la bande de transmission est réutilisée. Les communications RF requièrent des points d'accès, tandis que le LiFi requiert seulement des éclairages à LED installés et ne consomme donc pas plus d'énergie que l'éclairage à LED. De plus, la norme IEEE 802.15.7 [1] prévoit l'utilisation de variateurs qui régulent l'intensité d'éclairage et la consommation d'énergie électrique. L'utilisation d'un variateur et du circuit de transmission Li-Fi doit garantir l'efficacité d'éclairage des lampes. En termes d'infrastructure, le remplacement d'ampoules d'éclairage pour les nouvelles ampoules à LED permettra de créer un réseau à double fonctionnalité: éclairage et communications. La lumière visible est si éloignée de la bande RF qu'aucune interférence n'est possible avec d'autres équipements fonctionnant sur le RF, ce qui permet l'utilisation du Li-Fi dans les hôpitaux (figure 1) et les avions, en toute sécurité. Le Li-Fi a une zone de communication qui se limite à la zone d'éclairage, ce qui restreint sa portée à cette zone, mais cela peut constituer également un avantage en ce qui concerne la sécurité. Les RF sont, par contre, nettement moins sensibles au bruit que le Li-Fi. Le Li-Fi vient donc en complément des communications RF, il ne se substitue pas à elles. Le tableau 1 présente une brève comparaison entre les communications RF et le Li-Fi et permet de faire ressortir les avantages et les inconvénients de chaque technique.

\section{Croisement de technologies}

Le Li-Fi est une technologie à mi-chemin entre les communications réseaux et l'éclairage. En se basant sur le modèle OSI (open system

Le Li-Fi était déjà dans les esprits vers 2000 , mais ce n'est qu'en 2005 que son développement prend forme. Il faut attendre 2010 pour que le nombre annuel de publications sur le sujet - brevets et articles - dépasse le seuil des 100 et 2015, pour qu'il dépasse les 1000 au total. Nous tenterons d'expliquer cet intérêt grandissant.

À la différence des Wi-Fi, Zigbee, LoRa et Bluetooth, le Li-Fi utilise la bande visible du spectre

\begin{tabular}{|l|c|c|}
\hline Spectre disponible & RF ET MICRO-ONDES & LI-FI \\
Complexité & $300 \mathrm{GHz}$ & $380 \mathrm{THz}$ \\
Couverture & Élevée & Faible \\
\hline $\begin{array}{l}\text { Interférence - bruit } \\
\text { Interférence électromagnétique } \\
\text { Consommation de puissance }\end{array}$ & Longue portée & $\begin{array}{c}\text { Limitée à } \\
\text { lnfrastructure }\end{array}$ \\
\hline
\end{tabular}

Tableau 1. Brève comparaison entre les communications RF et Li-Fi. 


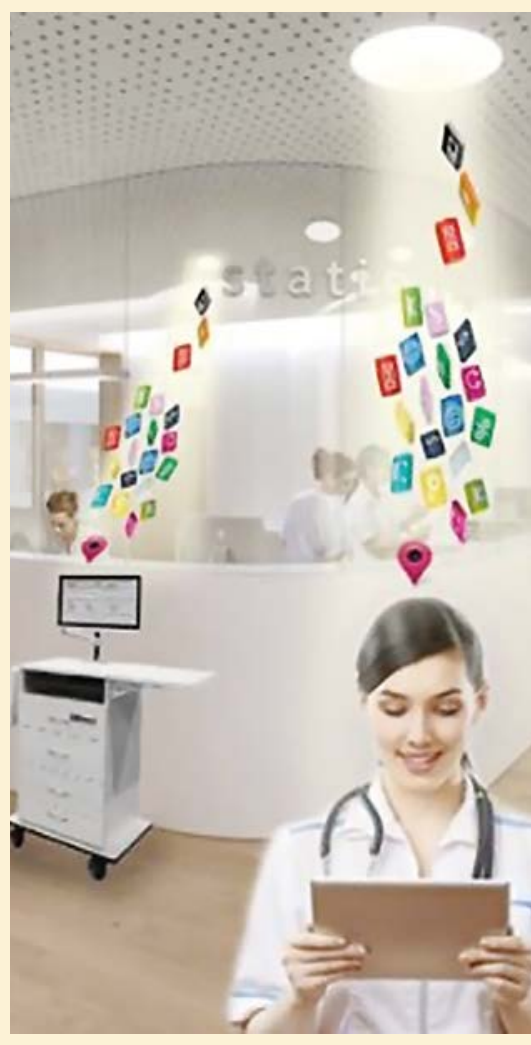

Figure 1. Système Li-Fi à l'hôpital

interconnection), une carte électronique Li-Fi contient une couche PHY pour moduler/démoduler le signal, le filtrer et l'amplifier (figure 2). Elle contient également une couche MAC pour effectuer la gestion et l'interprétation de données. La carte fait un (ou plusieurs) pont qui relie l'information provenant d'une MAC sans Li-Fi et d'une MAC Li-Fi pour que cette information puisse être relayée par la lampe. Il est possible d'accéder au signal à transmettre en passant par une de ces techniques: (a) fibre optique, (b) PoE (power over ethernet), (c) CPL (courant porteur en ligne), ou encore (d) 4G-5G. Afin d'exploiter le Li-Fi à haut débit, l'accès par fibre optique est recommandé.

\section{L'émetteur}

L'émetteur d'un transceiver Li-Fi est la LED dont la fonction d'éclairage garantit plus de 50000 heures d'utilisation tout en réduisant la consommation énergétique de $80 \%$. Les LED les moins chères sont les pc-LED (phosphor converted) qui consistent en un chip LED bleue d'InGaN qui pompe une couche de phosphore YAG. Le phosphore convertit une partie de la lumière bleue en vert, jaune et rouge; un mélange des intensités des couleurs primaires permet d'obtenir du blanc froid, du blanc neutre ou du blanc chaud tout en conservant la meilleure efficacité. Les LED à trois couleurs (RVB), connues comme multi-chip LED, sont sensiblement plus chères mais leur bande passante est plus large.

\section{Le récepteur}

Le récepteur se compose de deux éléments clefs : la photodiode (PD) et la lentille collectrice de lumière. Le choix de conception de la lentille dépend du choix de la PD. Une PD à avalanche présente un gain élevé mais elle est également plus sensible au bruit quantique du fait du mécanisme d'avalanche. Les PD de type PIN sont plus stables aux températures élevées, ainsi qu'aux flux lumineux intenses et leur coût est moindre. Pour une application de bas débit, une PD PIN est une bonne option; en revanche pour une application haut débit, une PD à avalanche est préférable. Le temps de réponse d'une PD est proportionnel à sa capacitance: plus la taille de la surface de détection est petite, plus la capacitance est petite et plus la réponse est rapide. En contrepartie, le flux lumineux est moins important et collecte donc moins de signal. On peut utiliser des lentilles pour booster la réception du signal en permettant aux capteurs d'augmenter le flux de lumière, ceci se traduit par (a) une augmentation du débit, (b) une augmentation de la portée du signal. Lors de la conception des lentilles, il faut tenir compte de la forme, car à cause de sa taille et de son volume, une lentille hémisphérique n'est pas intégrable dans les dispositifs de communication mobile. Il faut également prendre en considération le volume total de la lentille et, in fine, le grossissement de l'image de la source émettrice.

\section{DE FAISCEAUX LC YAG FIXES TECHSPEC ${ }^{\oplus}$}

Conception Compacte LC

(Low Cost - Prix Économique) pour Intégration OEM

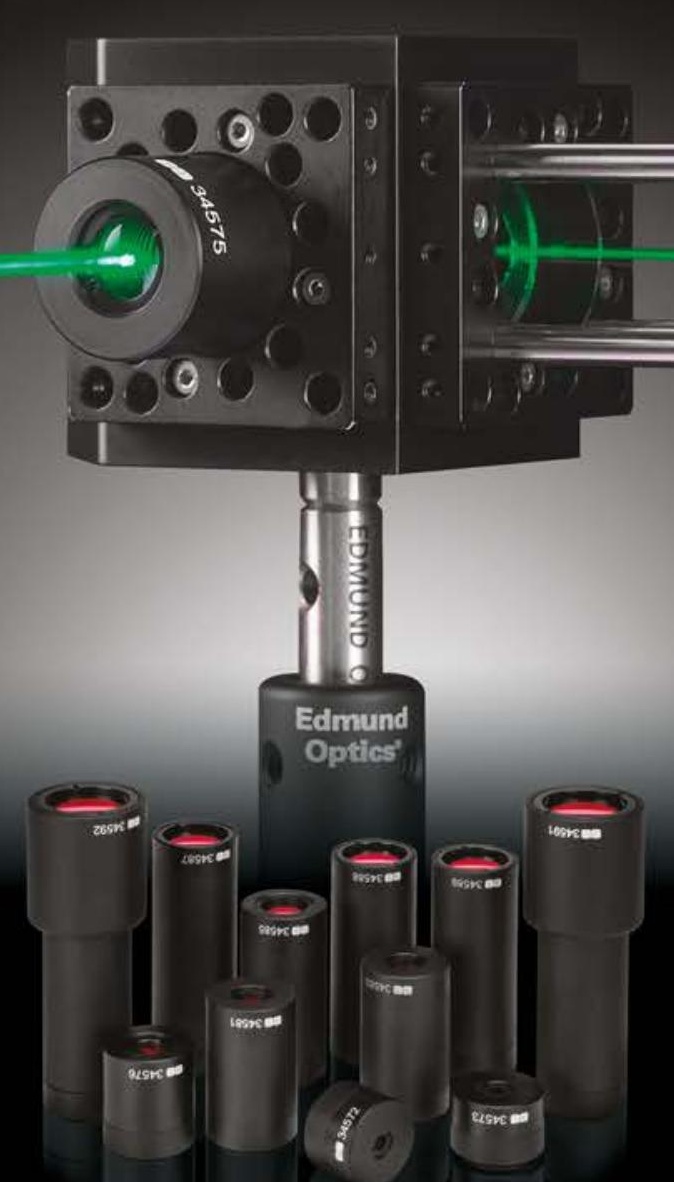

- Performance à la Limite de la Diffraction

- Conçus pour Longueurs d'Onde YAG

- Conception Galiléenne à 2 Éléments

- Grossissements et Longueurs d'Onde Personnalisés Disponibles

Rendez-nous visite:

LASER PHOTONICS

Munich, Kiosque B1.511

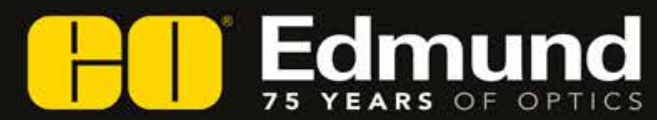




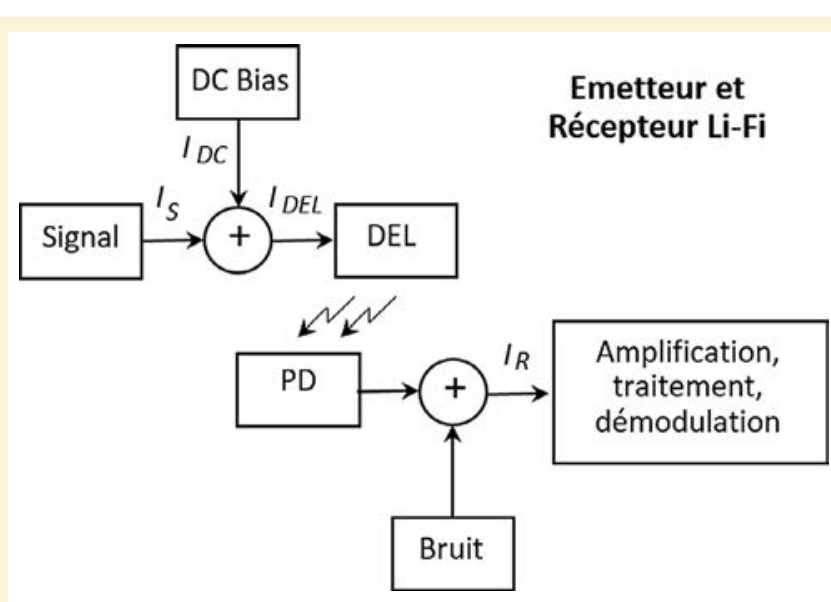

Figure 2. Schéma basique du système Li-Fi. $I_{D C}$ : courant bias, $I_{S}$ : courant du signal, $I_{L E D}$ : courant d'alimentation de la LED, $I_{R}$ : courant du signal. (a)
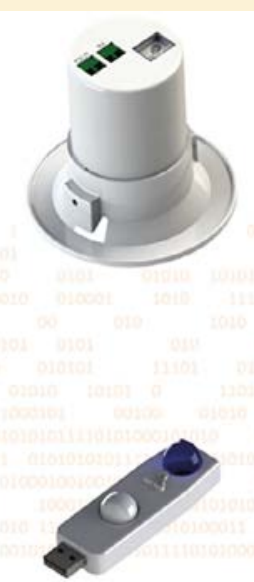

Figure 3. (a) Système d'internet Li-Fi bidirectionnel à bas coût à 10 Mbps. Lampe/transceiver et transceiver USB symétriques. (b) Cas applicatif: le capteur est intégré dans la tablette.

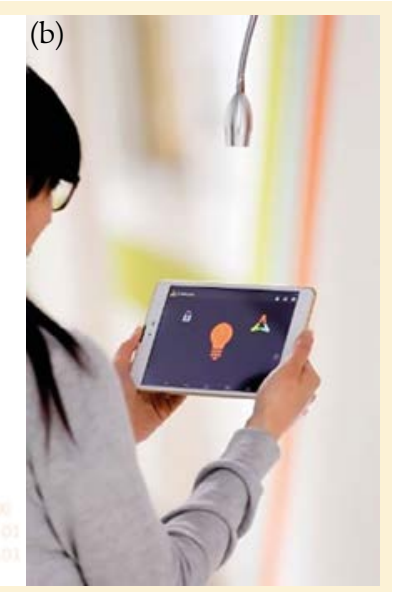

(1)

\section{Modulation et démodulation}

D’après le standard IEEE 802.15.7 [1], le bas débit pour une couche PHY I est modulé avec une modulation OOK (on offkeying) ou VPPM (variablepulse position modulation). Le débit atteint $100 \mathrm{~kb} / \mathrm{s}$ dans le premier cas pour une fréquence d'horloge de $200 \mathrm{kHz}$ et de $266,6 \mathrm{~kb} / \mathrm{s}$ avec une fréquence d'horloge de $400 \mathrm{kHz}$ pour la modulation VPPM. Lorsqu'on utilise une couche PHY II, une modulation OOKà fréquence d'horloge de 120 $\mathrm{MHz}$ permet de transmettre $96 \mathrm{Mb} / \mathrm{s}$. L'utilisation d'une modulation VPPM avec fréquence d'horloge de 7,5 MHz permet d'atteindre $5 \mathrm{Mb} / \mathrm{s}$. La couche PHY III est réservée à la modulation colour shift keying (CSK) qui requiert des LED multi-chip; elle permet de transmettre $96 \mathrm{Mb} / \mathrm{s}$ en mode 16-CSK avec une fréquence d'horloge de $24 \mathrm{MHz}$. Des taux plus élevés ont été expérimentalement établis avec une modulation d'amplitude en quadrature (QAM). Celle-ci est accompagnée d'un processus de codage de signaux numériques par répartition en fréquences orthogonales sous forme de multiples sous-porteuses (OFDM: orthogonal frequency division multiplexing). D'autres schémas, par exemple celui de modulation d'amplitude et phase sans porteuse (CAP), ont atteint des débits supérieurs à $1 \mathrm{~Gb} / \mathrm{s}$ [3]. Il reste encore à orienter la recherche sur l'optique de réception pour permettre d'élargir la portée de détection.

\section{Solutions Li-Fi}

Il existe diverses applications du Li-Fi qui requièrent des transmissions à bas débit comme par exemple la géolocalisation, $\mathrm{l}^{\prime}$ internet des objets connectés (IoT) et des communications bidirectionnelles qui ne relèvent pas du haut débit. En ce qui concerne la géolocalisation, qu'elle soit dans des espaces intérieurs ou extérieurs, elle s'appuie sur un réseau de balises. Le Li-Fi peut convertir l'éclairage intérieur et extérieur en relais de communications [4] et intégrer un dispositif externe de réception au smartphone ou utiliser ses capteurs comme le ALS (ambient light sensor) ou la caméra pour détecter le signal [5]. La gamme de solutions d'Oledcomm comprend le bas débit unidirectionnel pour la

\section{RÉFÉRENCES}

[1] LAN/MAN Standards Committee, IEEE standard for local and Metropolitan area networks Part 15.7: Short-Range Wireless Optical Communication Using Visible Light. IEEE (New York) 2011.

[2] D. Karunatilaka, F. Zafar, V. Kalavally, R. Parthiban, Led based indoor visible light communications: State of the art, IEEE Commun. Surv. Tut., 17 (3), 1649-1678 (2015).

[3] Y. Wang, X. Huang, L. Tao, J. Shi, N. Chi, 4.5-Gb/s RGB-LED based WDM visible light communication system employing CAP modulation and RLS based adaptive equalization, Opt. Express, 23 (10), 13626-13633 (2015).

[4] S. Topsu, C. Domínguez, H. Pérez, L. Chassagne, H. Guan, M. Rozenblat, J. García-Márquez, Transmission de données et géolocalisation avec des lampes AC à LED modulées, Colloque francophone CMOI-FLUVISU, Le Mans, France (2017). https://hal.archives-ouvertes.fr/ hal-01497884/document

[5] J. Green, H. Pérez-Olivas, S. Martínez-Díaz, J. García-Márquez, C. Domínguez-González, R. Santiago-Montero, H. Guan, M. Rozenblat, S. Topsu, VLC-beacon detection with an under-sampled ambient light sensor, Opt. Commun., 397, 122-128 (2017). 